Article

\title{
Insights into Ferric Leaching of Low Grade Metal Sulfide-Containing Ores in an Unsaturated Ore Bed Using X-ray Computed Tomography
}

\author{
Katherine J. Dobson ${ }^{1,2}$, Sue T. L. Harrison ${ }^{3, *}$, Qingyang Lin ${ }^{4}$, Aine Ní Bhreasail ${ }^{5}$, \\ Marijke A. Fagan-Endres ${ }^{3}$, Stephen J. Neethling ${ }^{4}$, Peter D. Lee ${ }^{1,6}$ and Jan J. Cilliers ${ }^{4}$ \\ 1 School of Materials, University of Manchester, Oxford Rd., Manchester M13 9PL, UK; \\ katherine.dobson@durham.ac.uk (K.J.D.); peter.lee@manchester.ac.uk (P.D.L.) \\ 2 Department of Earth Sciences, Durham University, Durham DH1 3LE, UK \\ 3 Centre for Bioprocess Engineering Research, Department of Chemical Engineering, \\ University of Cape Town, Private Bag X6, Rondebosch 7701, South Africa; marijke.fagan@uct.ac.za \\ 4 Department of Earth Science and Engineering, Royal School of Mines, Imperial College London, \\ London SW7 2AZ, UK; q.lin11@imperial.ac.uk (Q.L.); s.neethling@imperial.ac.uk (S.J.N.); \\ j.j.cilliers@imperial.ac.uk (J.J.C.) \\ 5 Department of Materials, Imperial College London, Prince Consort Rd., London SW7 2BP, UK; \\ ainenib@gmail.com \\ 6 Research Complex at Harwell, Rutherford Appleton Laboratories, Harwell, Oxfordshire OX11 0FA, UK \\ * Correspondence: sue.harrison@uct.ac.za; Tel.: +27-21-650-2759
}

Academic Editor: M. Thaddeus Ityokumbul

Received: 22 February 2017; Accepted: 16 May 2017; Published: 22 May 2017

\begin{abstract}
The distribution of the metal-bearing mineral grains within a particulate ore prepared for leaching, and the impact of this spatial heterogeneity on overall extraction efficiency is of key importance to a mining industry that must continuously target ever-reducing grades and more complex ore bodies. If accessibility and recovery of the target minerals is to be improved, a more detailed understanding of the behaviour of the system must be developed. We present an in situ analysis using X-ray computed tomography to quantify the rates of volume reduction of sulfide mineral grains in low grade agglomerated copper bearing ores during a miniature laboratory scale column leaching experiment. The data shows the scale of the heterogeneity in the leaching behaviour, with an overall reduction of sulphide mineral grains of $50 \%$, but that this value masks significant $\mathrm{mm}^{3}$ to $\mathrm{cm}^{3}$ scale variability in reduction. On the scale of individual ore fragments, leaching efficiency ranged from $22 \%$ to $99 \%$. We use novel quantitative methods to determine the volume fraction of the sulfide that is accessible to the leachate solution.
\end{abstract}

Keywords: X-ray tomography; leaching; chalcopyrite

\section{Introduction}

The continued demand for commodity metals such as copper requires extraction from increasingly diminishing grades of ore, so maximising the efficiency of recovery from low grade (typically $\sim 0.1 \%-0.8 \%$ ) ores in overall and energetic terms is of significant economic and environmental interest. Ideally these low-grade ores are processed to maximise metal recovery, leaving a waste rock with reduced environmental impact. Where waste rock is dumped without the recovery of the low concentration metals, understanding of natural leaching within the dump is equally as important for ensuring minimal environmental impact [1]. Biologically enhanced heap leaching is a key technology for the economically viable recovery of metals from high-volume low grade sulfide ores, and is used for the extraction of copper, zinc and nickel, amongst others [2-5]. Run-of-mine ore or crushed and 
agglomerated ores are stacked into heaps on impervious pads designed for solution recovery, typically with aeration systems provided below the heap. These heaps may be inoculated by naturally occurring micro-organisms, either during agglomeration or through irrigation. Irrigation is typically from drip irrigators spread over the top of the heap at regular intervals. The metals solubilised by ferric iron and acid lixiviants are reclaimed from the pregnant leach solution (PLS), prior to recirculation of the solution to the heap. Typical metal extraction from the heap is $60 \%$ to $90 \%$ over one to two years $[3,6]$. Analysis of the PLS typically shows that $50 \%$ to $60 \%$ of the metal is released during an initial period of rapid leaching, followed by much slower release. The decrease in recovery rates is attributed to depletion of easily soluble minerals as well as reduced accessibility of the metal-bearing mineral grains to the micro-organisms and leachate solution, but may also be controlled by changes in reaction kinetics or passivation of the reactive surfaces through precipitation or other processes [7]. While the mechanisms controlling leaching kinetics of milled ores with liberated sulfide grains can be experimentally determined by tank leaching studies [8-10], the leaching behaviour of equivalent sulfide grains within an ore fragment in a heap is less well characterised.

In order to maximise the recovery efficiency of metal from low-grade ore, it is necessary to both increase yields and reduce leach durations; attainment of this requires an improved understanding of the contacting of the leach agents and mineral surface, informing leach behaviour in the heap at all length scales, especially on the $\mathrm{mm}^{3}$ to $\mathrm{cm}^{3}$ scale, thus linking macro and micro scale understanding. This requires both an understanding of fluid flow and of mineral grain distribution in the ore bed of the heap.

Traditional methods for analysing changes in sulfide content on the fragment scale are destructive, two dimensional and spatially limited. These cannot provide in situ observations. The distribution of sulfide grains within the low-grade ore and the behaviour of individual grains as a function of time during leaching is therefore poorly quantified. Simplified models have been provided to predict metal dissolution from the ore. Owing to their limited mechanistic basis, their application is limited to bulk predictions or to systems very similar in geometry and ore bed characteristics to those used in model generation, providing limited value.

X-ray micro computed tomography $(\mu \mathrm{CT})$ is a non-destructive 3D imaging technique that permits visualisation of the internal structure within the sample [11,12]. It can therefore provide an invaluable method for assessing mineralogical and spatial controls on the spatial heterogeneity of the leaching process within a column, with potential extension to predict the maximal expected leachability of the material in larger scale heaps. Samples can be imaged in situ in small scale laboratory experiments, allowing a consistent ore volume to be monitored over the course of a leaching experiment without disturbing the macro-scale configuration of the packed bed of ore fragments or agglomerated material. To date X-ray $\mu \mathrm{CT}$ application to heap leaching research has been successfully demonstrated for the study of bed voidage evolution [13], ore fracturing [14-16], mineral liberation [17] and mineral leach kinetics [18-21].

In this study, the latest X-ray computed micro tomography $(\mu \mathrm{CT})$ techniques are used to perform in situ visualisation and quantification of the mineral sulphide (MS) grains and gangue fractions, as well as assessment of the mineralogy (in this sample for some phases only), porosity and fracture networks within individual ore fragments, and the ore bed, as they undergo leaching in a small-scale laboratory column experiment. Time integrated X-ray $\mu \mathrm{CT}$ is exploited to quantify changes in the volume fraction of sulfide mineral during leaching, and to illustrate how $\mu \mathrm{CT}$ can be used to investigate key spatial parameters that control the behaviour of sulfide grains during leaching of low grade agglomerated ores and thereby interrogate the degree of leaching behaviour variability across different length scales.

\section{2. $\mu \mathrm{CT}$ of Laboratory Scale Columns}

In $\mathrm{X}$-ray $\mu \mathrm{CT}$ imaging the sample is mounted onto a rotating stage between the $\mathrm{X}$-ray source and a detector, and a series of radiograph images (projections) are collected as the object rotates within a stationary X-ray beam (Figure 1A). Projections are collected at equal angular intervals during a $360^{\circ}$ 
rotation. Each X-ray that passes through the sample has a characteristic energy, and interacts with the mineral phases found along the beam path. This interaction (attenuation) is largely controlled by the atomic number; with denser materials attenuating (absorbing) more strongly. Each pixel of a single projection represents the net $\mathrm{X}$-ray attenuation along a particular beam path. As the sample rotates, the mineralogy along the beam path changes and each projection records a different $X$-ray intensity map. Mathematical algorithms are then used to reconstruct a 3D map, computing the attenuation coefficient of every voxel $\left(1\right.$ pixel $\left.^{3}\right)$ in the scan volume.

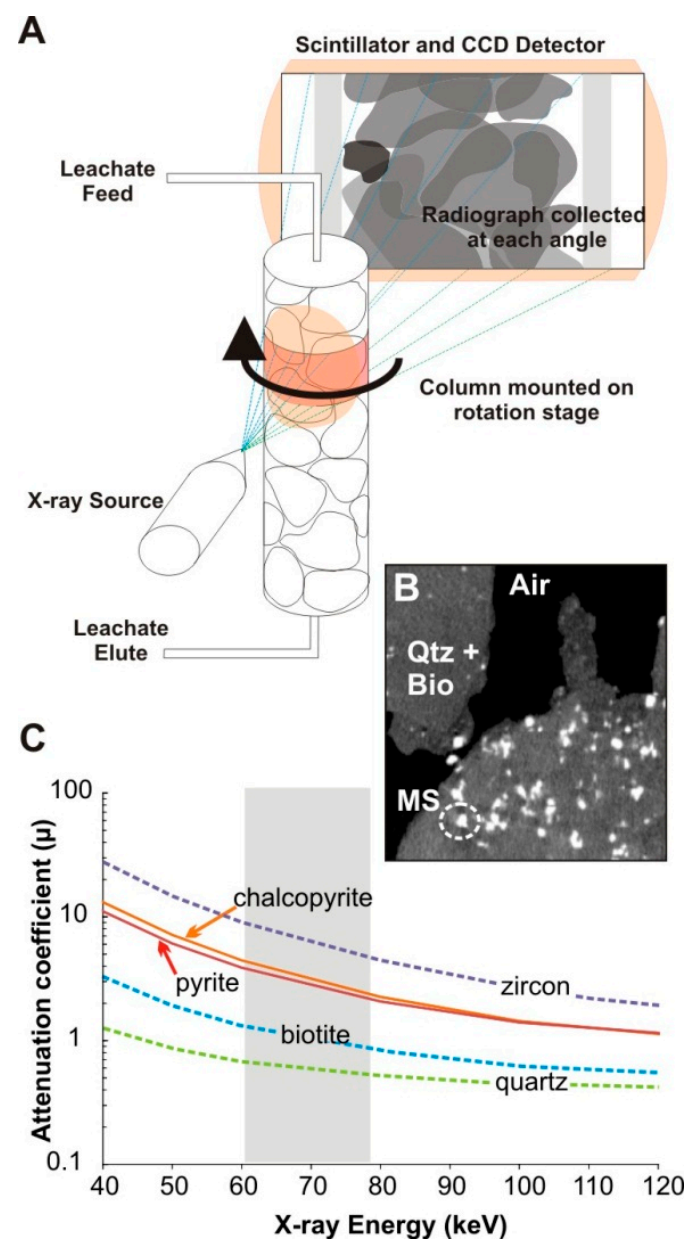

Figure 1. (A) Schematic showing the experimental set-up and the principals of $\mu \mathrm{CT}$ scanning; (B) A small area of a single 2D slice through the 3D column volume, showing the difference between the air (black), gangue (grey) and mineral sulfide grains (MS grains) (white); (C) The difference in attenuation of the different MS and gangue phases in the sample.

$\mu \mathrm{CT}$ was performed using a laboratory Phoenix-v I tome system, with an accelerating voltage of $100 \mathrm{kV}$ and a current of $70 \mu \mathrm{A}$. Six hundred projections were collected at $0.6^{\circ}$ angular increments on a $1015 \times 512$-pixel detector. All scans were performed at the same resolution (voxels of approximately $33 \mu \mathrm{m} \times 33 \mu \mathrm{m} \times 33 \mu \mathrm{m}$ ). Filtered back projection reconstruction was performed using the Phoenix proprietary software. Visualisation and quantification were performed using standard functions built into ImageJ-Fiji [22], and Avizo ${ }^{\odot}$. The reconstructed data are presented as $2 \mathrm{D}$ slices (vertical and horizontal) through greyscale volume data or as 3D renders of phase surfaces (Figure 2). 

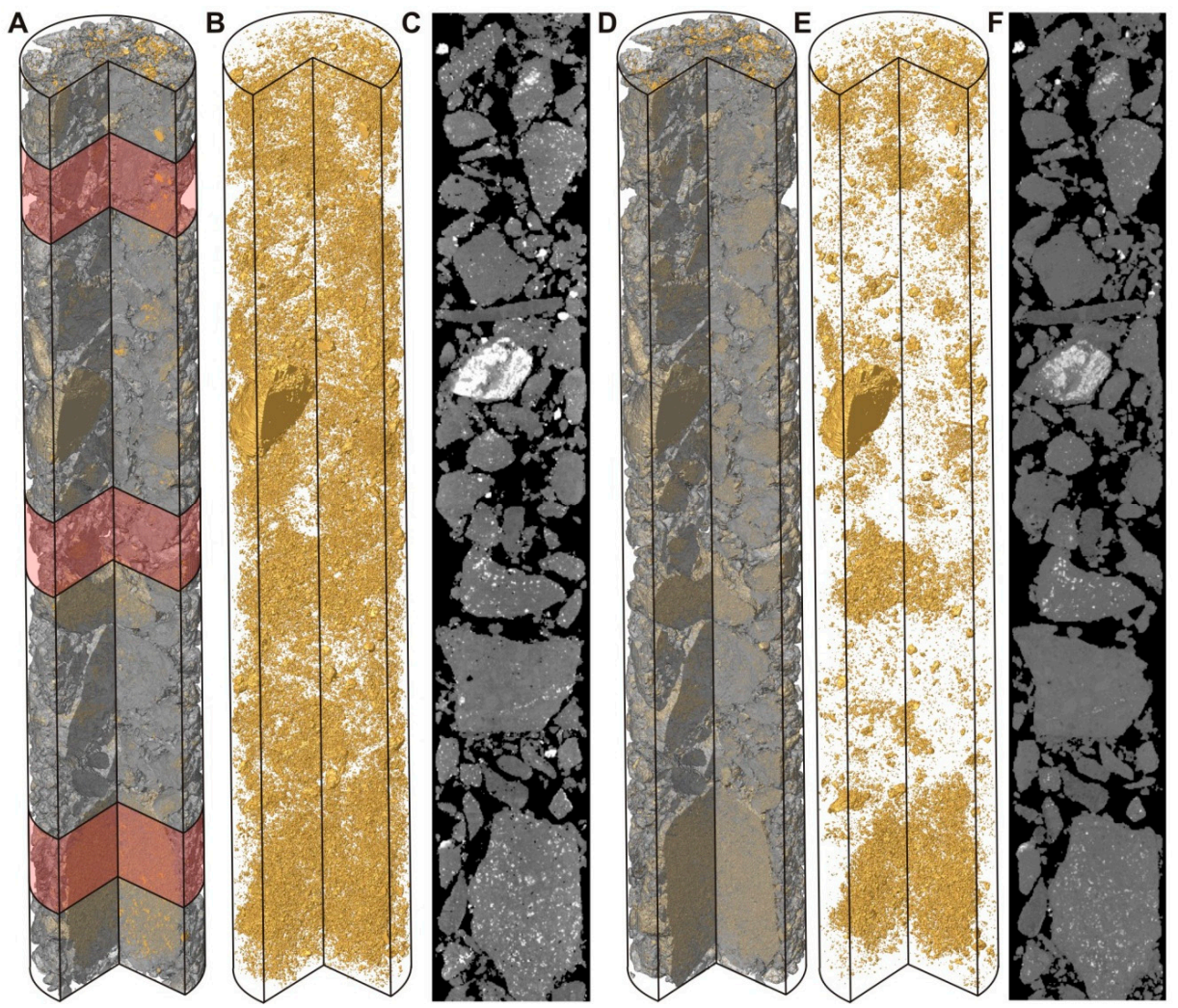

Figure 2. 3D render of the column $\mu \mathrm{CT}$ data (A) Day 0 render of ore (grey) and MS grains (gold); (B) Day 0 render of MS grains (gold); (C) 2D slice through the column at Day 0; (D) Day 192 render of (grey) and MS grains (gold); (E) Day 192 render of MS grains (gold); (F) 2D slice through the column at Day 192. The location of the three sub volumes used for time series scanning are shown in red.

The study was conducted using a low-grade ore from the Escondida deposit, containing $0.69 \mathrm{wt} \%$ copper (primarily as chalcopyrite $(0.5 \mathrm{wt} \%)$, chalcocite $(0.2 \mathrm{wt} \%)$, covellite $(0.3 \mathrm{wt} \%)$ and bornite $(0.1 \mathrm{wt} \%)), 2.95 \mathrm{wt} \%$ iron (including that from pyrite $(4.0 \mathrm{wt} \%))$, and $2.02 \mathrm{wt} \%$ sulfur [21,23]. Detailed analysis of the sulfide chemistry on a grain scale is beyond the scope of this study, and cannot be resolved by non-destructive techniques at this time. The purpose of this study was to quantify leaching induce changes in sulfide and gangue distribution in situ, during leaching, without disruption of the sample. The ore was acid agglomerated [24], loaded into a glass leaching column (length $=220 \mathrm{~mm}$; internal diameter $=26 \mathrm{~mm}$ ), and allowed to air dry. Fines were negligible [21]. The leachate solution, containing $0.1 \mathrm{M} \mathrm{H}_{2} \mathrm{SO}_{4}$ with $5 \mathrm{~g} / \mathrm{L}$ ferric iron $(\mathrm{emf}=668 \mathrm{mV}$, $\mathrm{Eh}=865 \mathrm{mV}, \mathrm{pH}=1.5)$, was gravity fed into the column at $\sim 4 \mathrm{~mL} / \mathrm{h}$ from a single drip emitter point source. The column was not inoculated with micro-organisms and was operated at ambient conditions. A 3D reconstruction of the entire column was acquired by concatenating $13 \mu \mathrm{CT}$ scans, and three sub volumes containing typical ore geometry and mineralogy were identified from the top (V1), middle (V2) and bottom (V3) of the column (Figure 2). The leachate was then allowed to percolate through the column. The column was removed periodically from the flow to allow the scanning of V1, V2 and V3 at 12 time points over a 192-day leaching period. These 36 scan volumes were then used to track evolution of the ore at three different locations along the column. The full column was scanned again at the end of the leaching period. No part of the column was disturbed at any point. On removal from the flow, the column was maintained in the vertical position and transported carefully to the scanner (in the same room) with no impact, vibration or other disturbance. All reasonable care was taken during handling. Ore particles do not show any significant change beyond that expected with ongoing irrigation of the column. Markers on the outer surface of the column were used to register (align) the images of V1, V2 
and V3 prior to quantitative analysis, and to check that there was no significant solids mass transfer (other than that facilitated by leaching) into or out of the volumes used for quantification. The glass tubing of the column was cropped from all images using a cylindrical mask.

\section{3. $\mu \mathrm{CT}$ as a Tool for Assessing Compositional and Behavioural Heterogeneity}

V1, V2 and V3 were selected to include representation of surface grains, large grains, small grains and seams within the bulk ore to enable behaviour of these to be assessed. $\mu \mathrm{CT}$ analysis is one of a family of tomographic tools, including magnetic resonance imaging (MRI) [25], positron emission tracking (PET) and positron emission particle tracking (PEPT) [26] and electrical resistance tomography (ERT) [27], that allow the comparison of identical ore volumes before and after leaching without disturbing the experiment. $\mu \mathrm{CT}$ allows the imaging of the solid phase in this manner in particular. It therefore allows the visualisation of the complex structural and mineralogical heterogeneity of the ore; and, most crucially, an assessment of how different sulfide grain volumes evolve over time during leaching. We have determined qualitative and quantitative information on the behaviour of the sulfide grains using the pre- and post-leaching scan volumes, and performed additional quantitative assessment using the time series analysis from V1, V2 and V3. The nature of the agglomerated ore can be seen in the 2D slices through the reconstructed data volumes (Figures 2 and 3 ). The bracketed numbers in the following discussion refer to typical examples of microstructures and mineral associations shown in Figure 3.
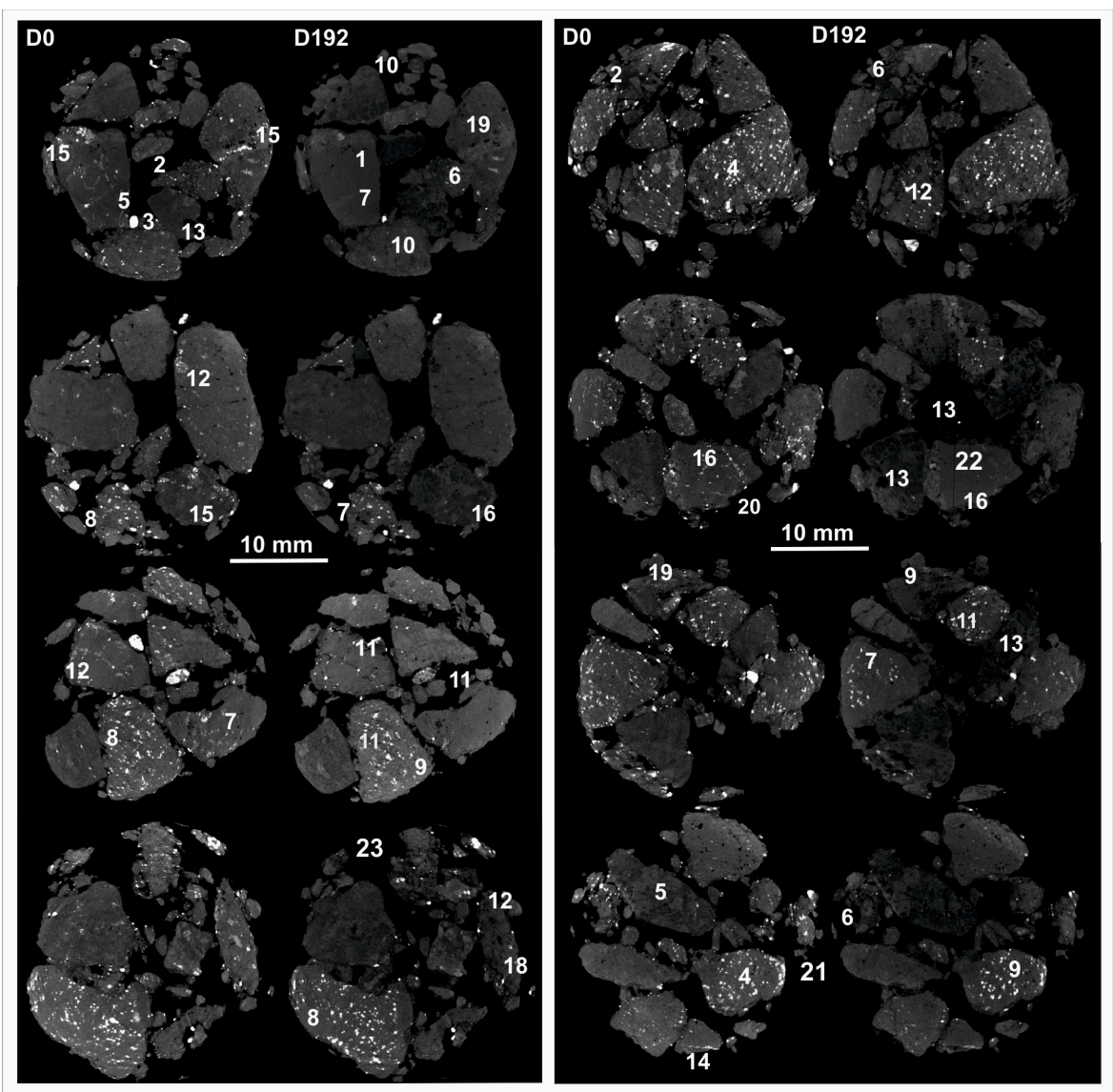

Figure 3. 2D micro-CT slices through the three volumes (V1-V3) at Day 0 and Day 192, showing the pre- and post-leaching ore fragment and MS grain geometries, and the changes to the internal structures observed in the column that are observed over time. The numbers correspond to the discussion in Section 3.1 and highlight key features and changes. All images are 2D slices in the radial (xy) plane $26 \mathrm{~mm}$ diameter. 


\subsection{Qualitative Observation of an Ore during Leaching}

Three main phases can be identified from their grey scale intensity (X-ray attenuation): air (black, low X-ray attenuation), gangue minerals (mid grey, mid X-ray attenuation), and grains with high metal content (pale grey to white, high X-ray attenuation) (Figure 1B). Both the gangue and metalliferous materials show local greyscale variability which corresponds to mineralogical or density variations. The calculated X-ray attenuation differences between pure specimens of the main $\mathrm{Fe}$ - and $\mathrm{Cu}$ - bearing sulfide phases (pyrite, chalcopyrite, chalcocite and covellite) are small (Figure 1C); hence mineral identification based on image greyscale intensity was not carried out in this case.

The material has been agglomerated to avoid partitioning of fines within the column. Very little non-agglomerated fine material was observed in the initial scan. All three volumes show variability in gangue and sulfide grain geometry, volume and distribution, as is expected in the ore bed for heap leaching. The gangue mineralogy can be subdivided into: large fragments of brighter, coherent homogenous material (Figure 3) (1); and areas of breccia containing angular to sub-angular fragments of the paler material (2); The darker breccia groundmass suggests that fine scale porosity (below image resolution) caused a reduction in the bulk density. The coherent fragments are often coated in a rind of the breccia groundmass (3) as intended through the agglomeration process; The coherent fragments typically have higher volume fraction sulfide (4) than the clasts (5) and groundmass (6) of the breccia; The sulfide grains are found as both fully enclosed or liberated grains (7); There are rare grains with much higher X-ray attenuation than the bulk of the metal-bearing phases. These grains are found both enclosed within regions of high metal content, and as isolated grains (8); and are accessory phases, possibly oxide phases.

The reduction in the total sulfide volume and sulfide grain number with increasing time of leaching is apparent in all slices, but there is significant spatial heterogeneity in the leaching efficiency. The breccia material generally shows a higher leaching efficiency (9); The more coherent ore fragments exhibit variable leaching behaviour: some show a high degree of leaching (10); others show very little change (11); and some exhibit internally variable leaching (12); Some of the larger liberated sulfide grains have been removed completely. In some instances, this appears to have occurred by leaching (13); whereas other grains may have dislocated from the region of study (14); Elsewhere the complex inter-grown nature of the sulfide phases is indicated by the variable and sieve like dissolution textures of some large sulfide grains (15), consistent with the differing leaching rates expected for pyrite, chalcocite and chalcopyrite.

Some coherent fragments contain clusters of highly attenuating, generally acicular to tabular accessory mineral phases (e.g., muscovite, chlorite, apatite, and amphibole etc.) (16). While the majority of the gangue and the oxide very highly attenuating phases (17) show little change during leaching, these crystals (18), and some smaller coherent fragments (19) have much lower post-leach attenuation. This implies preferential leaching of highly attenuating elements or more probably the partial or complete dissolution of the accessory phases, leaving high porosity regions within the rock fragments.

Fracture of the ore during primary crushing has, in rare cases, exploited narrow continuous planar sulfide bearing veins (20) leaving sulfide rich zones exposed along the surfaces of the coherent fragments. However, the more common mineralised veins contain larger more discontinuous, sometimes isolated, sulfide grains (21) and run through the interiors of fragments. Both forms of vein hosted sulfide are found as conjugate pairs in some fragments. In almost all cases the veins have been leached very efficiently (22). The initial ore has rare small fracture networks that may permit deeper fluid penetration. These are found in both the coherent and brecciated regions and seen to widen during leaching (not shown). Other fractures visible in the post-leaching images suggest that many of the fracture networks may be beyond image resolution $(33 \mu \mathrm{m})$ until they are widened by the leachate.

The pre- and post-leaching volumes showed movement and rotation of some fragments with respect to their neighbours and minor transport of darker agglomerated material (23) and some vertical compaction. Percolation of leachate fluids through the column is expected to evolve through time 
where the external (inter fragment) and internal (within fragment) porosity and fracture networks are affected by the leaching process.

\section{Quantitative Assessment}

Inter-particle porosity was segmented using a threshold value determined from the local minima between the air and gangue peaks in the greyscale intensity histogram. This method identifies only the largest internal pores within the fragments (Figure 3). Assessment of the full intra-fragment porosity was not achievable using the resolution suited to these typical laboratory experimental scanning conditions, but binary segmentation results (ore $=1$, not ore $=0$ ) were used to perform preliminary quantification of the ore and the inter-particle pore network.

The grains of the highly X-ray-attenuating accessory phases and the volumetrically dominant sulfide phases (hereafter termed MS grains) were segmented using a simple automatic threshold criterion multilevel thresholding technique. The thresholding segments all particles of pyrite, chalcopyrite and the highly attenuating oxides from the gangue minerals. A series of standard 2D and 3D image filtering techniques were tested, but all removed a significant and variable number of MS grains that could be tracked through time (i.e., are not noise), so no noise reduction filters were applied before segmentation. The segmentation yields a second binary volume ( $M S=1$, non-MS $=0$ ). While some of the small particles segmented using this method will be image noise, most will be sub-voxel sized MS grains. Image noise, partial volume effects and volume reduction mean that small particles were unlikely to be quantified accurately (not better than $\pm 5 \%$ ) in all time points [28] when working with sub volumes of the data and on individual MS grains. We therefore applied a volumetric filter used to discard all grains with volumes below of 5 voxels $\left(\sim 180,000 \mu^{3}\right)$. All volume assessments are presented as volume fractions. All voxels not labelled as metal sulfide (MS grains) or pore after both segmentation methods were defined as gangue.

\subsection{Column Scale Parameters and Bulk Leaching Behaviour}

From the $\mu \mathrm{CT}$ images, the column was estimated to contain $\sim 67 \%$ ore by volume. This is consistent with voidages of $38 \%$ and $41 \%$, estimated gravimetrically and by tomographic imaging, for the packing of the same ore into a similar column for MRI studies [29]. At Day 0, the ore was estimated to contain $6.9 \mathrm{vol} \% \mathrm{MS}$ from the $\mu \mathrm{CT}$ images. This calculated volume is somewhat higher than is expected from a bulk mineralogical analysis (the ore contains a total of $\sim 4.0 \mathrm{wt} \%$ pyrite, $\sim 0.5 \mathrm{wt} \%$ chalcopyrite, and a total of $\sim 5.4 \mathrm{wt} \%$ MS phases (i.e., all sulfide and oxide phases), but includes a single very large MS grain found within the column (Figure 2). This single particle makes up $\sim 1.5 \mathrm{vol} \%$ of the total ore. If this grain is not included in the calculation, the MS volume fraction ( 5.4 vol \%) is in good agreement with the known composition. The MS volume fraction reduces from $6.9 \mathrm{vol} \%$ (Day 0 ) to $4.0 \mathrm{vol} \%$ (Day 192) (Figure 2): a reduction of $41.9 \%$ over the leaching period. The total ore volume shows minimal change over the same period $(2 \%)$. Discounting the single large grain from both the pre- and post-leaching calculations generates a more representative bulk MS volume reduction of 48.2\% (5.4\% Day 0; $2.8 \%$ Day 192$)$.

Spatial heterogeneity of the MS grain distribution and leaching efficiency is visible in the full column data, suggesting local controls on leaching may operate at a local $(1-30 \mathrm{~mm}) \mathrm{scale}$. It is well recognised that spatial heterogeneity of the mineral grain distribution and variation in fluid distribution across the heterogeneous packing will contribute to such spatial variation. We use the three sub-volumes of the column to assess the magnitude of this localised variability.

\subsection{Volume \& Fragment Scale Behaviour}

Volume renders of the MS grains in V1, V2 and V3 are shown in Figure 4. The initial volume fraction MS is highly variable: $1.65 \%$ in V1, 3.06\% in V2, and 3.69\% in V3 (Table 1), and the volume reduction increases down the column, from $34.12 \%$ (V1) to $52.71 \%$ (V3). The average of the V1, V2 and V3 volume reductions (45.8\%) is in good agreement with the bulk value determined on the entire 
column (41.9\%). Macro scale quantification therefore hides significant local variability in leaching. Image registration ensured that the same volume of ore was scanned at each time point, but local rotation of the more coherent fragments, small scale changes in the fine-grained breccia groundmass and vertical compaction have been identified (Figure 2). However, the total volume of the ore and the volume fraction of the column that is accessible to liquid in each volume varies very little over the time period of 192 days ( $30.54 \%$ to $37.12 \%$ for the latter) (Table 1 ).

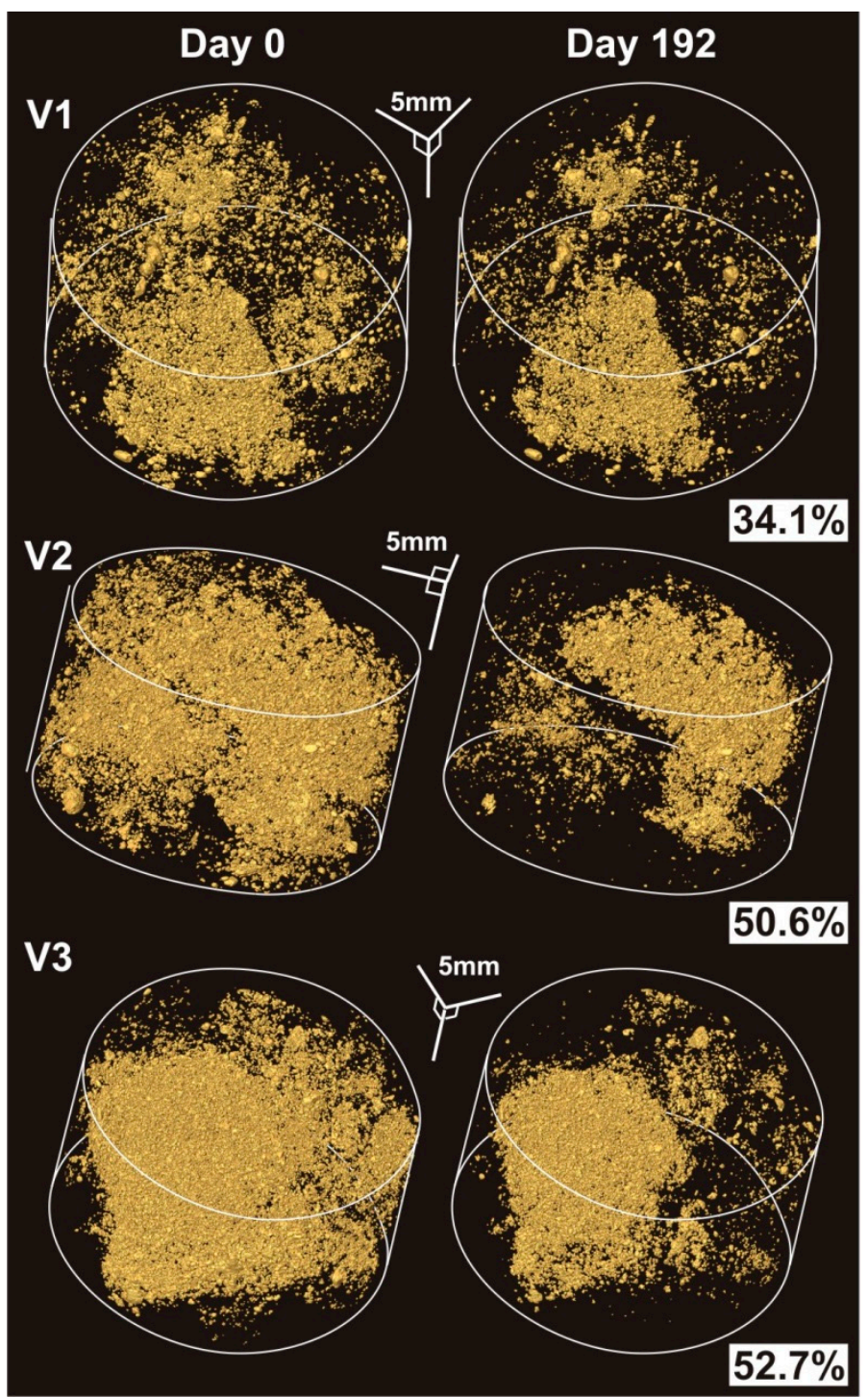

Figure 4. 3D render of the MS grains at Day 0 and Day 192, showing the overall reduction during leaching. MS grains are rendered in gold. The gangue has been rendered invisible. Only MS grains with volume $>5$ voxels are shown. The surfaces have been down sampled by a factor of 2 in all dimensions to improve clarity of the figure. The outline of the column volume is shown in white. All scale bars are $5 \mathrm{~mm}$. See Figure 2 for the location of the scan within the column.

The time series allow us to assess the application of the quantitative techniques for determination of the rate of MS volume reduction. All volumes are within a single column and experience similar leaching conditions provided through the irrigant; however, some spatial variation in leaching conditions and irrigant flow may be expected owing to variations in distribution of liquid flow across the unsaturated ore bed in the column. The temporal volume reduction across scan volumes is shown in Figure 5. This study focussed on applying quantitative techniques to assess the magnitude 
of MS volume reduction and the scale of leaching heterogeneity, not the specific leaching conditions. To assess the ability of the $\mu \mathrm{CT}$ images to record the response to a change in system conditions, the temperature of the column was increased from $23{ }^{\circ} \mathrm{C}$ to $60^{\circ} \mathrm{C}$ on Day 64 using heating tape, to simulate conditions within industrial scale heaps. V1 and V2 show negligible (V1) to very low volume reduction rate (V2) during the low temperature portion of the experiment (Day 1-60) followed by a faster volume reduction rate once the temperature had been increased, in accordance with the expected impact of temperature on leach rate of sulfidic minerals, especially chalcopyrite. V3 shows a similar reduction rate at the high temperature as seen in V2, but more variability in the early stage data at ambient temperature.

Table 1. Volumetric changes observed.

\begin{tabular}{|c|c|c|c|c|c|c|}
\hline & \multicolumn{2}{|c|}{ V1 } & \multicolumn{2}{|c|}{ V2 } & \multicolumn{2}{|c|}{ V3 } \\
\hline Volume of ore (voxels) & $97,733,700$ & $95,388,930$ & $98,112,370$ & $99,623,260$ & $104,996,300$ & $103,069,400$ \\
\hline Number of MS grains * & 12,112 & 8474 & 20,929 & 7859 & 29,844 & 14,093 \\
\hline Volume of MS (voxels) * & $1,608,660$ & $1,034,291$ & $2,997,445$ & $1,505,130$ & $3,878,633$ & $1,800,546$ \\
\hline Volume of MS $\left(\mathrm{mm}^{3}\right)$ & 57.81 & 37.17 & 10772 & 52.09 & 139.39 & 64.71 \\
\hline Pore volume (voxels) & 1949 & 2025 & 1882 & 1873 & 1665 & 1764 \\
\hline Connected Pore vol. $\left(\mathrm{mm}^{3}\right)$ & 1946 & 2024 & 1879 & 1872 & 1661 & 1762 \\
\hline Volume $\%$ connected pore & $35.6 \%$ & $37.1 \%$ & $34.8 \%$ & $34.3 \%$ & $30.5 \%$ & $32.2 \%$ \\
\hline$\%$ Change in Pore Volume & \multicolumn{2}{|c|}{$-4.23 \%$} & \multicolumn{2}{|c|}{$1.2 \%$} & \multicolumn{2}{|c|}{$-5.5 \%$} \\
\hline \multicolumn{7}{|l|}{ Accessible } \\
\hline Reduction in MS & \multicolumn{2}{|c|}{$43.42 \%$} & \multicolumn{2}{|c|}{$84.10 \%$} & \multicolumn{2}{|c|}{$84.86 \%$} \\
\hline$\%$ leaching & \multicolumn{2}{|c|}{$67.31 \%$} & \multicolumn{2}{|c|}{$74.89 \%$} & \multicolumn{2}{|c|}{$56.08 \%$} \\
\hline \multicolumn{7}{|l|}{ Directly Accessible } \\
\hline Volume MS (mm³) & 7.18 & 4.57 & 10.95 & 0.61 & 1500 & 2.40 \\
\hline Volume \% MS & $0.20 \%$ & $0.13 \%$ & $0.31 \%$ & $0.02 \%$ & $0.40 \%$ & $0.06 \%$ \\
\hline Fraction MS & $12.42 \%$ & $12.30 \%$ & $10.16 \%$ & $1.13 \%$ & $10.76 \%$ & $3.72 \%$ \\
\hline Volume $\%$ removed & \multicolumn{2}{|c|}{$0.07 \%$} & \multicolumn{2}{|c|}{$0.29 \%$} & \multicolumn{2}{|c|}{$0.33 \%$} \\
\hline Reduction in MS & \multicolumn{2}{|c|}{$34.79 \%$} & \multicolumn{2}{|c|}{$94.52 \%$} & & \\
\hline \% leaching & & & & & & \\
\hline Indirectly Accessible & & & & & & \\
\hline Volume MS $\left(\mathrm{mm}^{3}\right)$ & 23.40 & 12.32 & 37.54 & 7.22 & 33.55 & 4.81 \\
\hline Volume \% MS & $0.78 \%$ & $0.59 \%$ & $1.68 \%$ & $1.29 \%$ & $2.41 \%$ & $1.55 \%$ \\
\hline$\% \mathrm{MS}$ & $47.10 \%$ & $54.57 \%$ & $54.98 \%$ & $85.52 \%$ & $65.17 \%$ & $88.85 \%$ \\
\hline Volume $\%$ removed & & & & & & \\
\hline Reduction in MS & & & & & & \\
\hline \% leaching & & & & & & \\
\hline
\end{tabular}

* All particles less than 5 voxels in volume have been ignored in these calculations, this equates to $1.4 \%$ of total M-phase voxels at Day 0, and $0.92 \% \pm 13 \%(2 \sigma)$ for Day 192. Uncertainty on each grain size fraction in the data set is $<<1 \%$ [28].

Inspection of the 2D slices through the column volume suggests that the V1, V2 and V3 averaged reductions hide spatial variability on the inter- and intra-fragment scale. All of the ore volume was used to generate binary segmented datasets of every MS grain in the volume at each time point. In addition, fragment scale behaviour was quantified by the definition of a series of regions, where sub-regions of each volume were manually (automation of this process was impossible because of the changes in the overall porosity and mineralogy present) divided into a number of fragment scale 
regions at Day 0. In the Day 0 mask definition, all masks were set so that the boundaries did not pass through large MS grains. Each region encloses one or more distinct coherent fragments. Each of the 28 regions (distributed across all three volumes, see Table 2) was used to generate ore and MS sulfide binary segmented datasets for more detailed analysis of the volume fraction MS in each region at through time. A second suite of regions was used to define a selection of the large liberated MS grains.

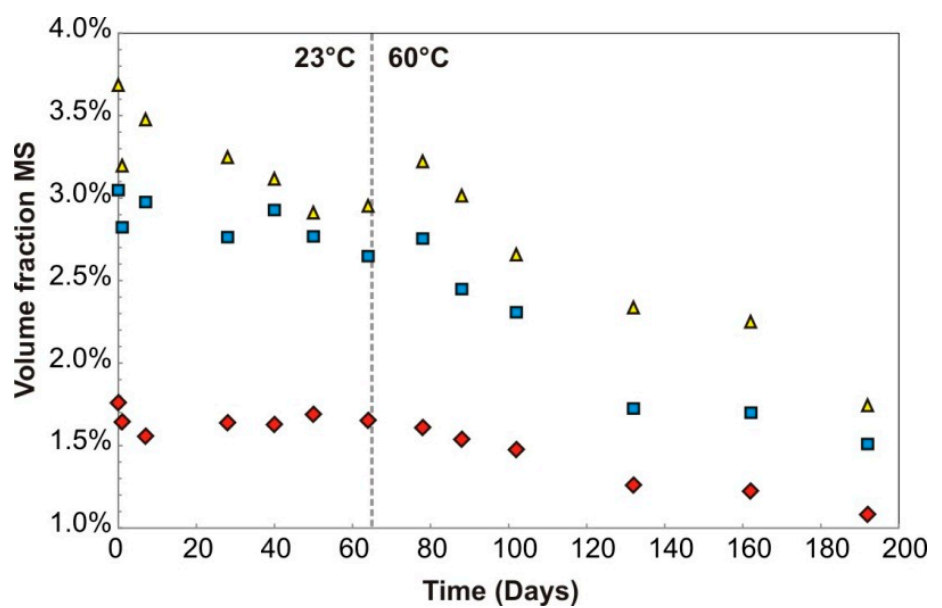

Figure 5. Reduction in MS grain volume fraction with time for V1 (red diamonds), V2 (blue squares) and V3 (yellow triangles). The slight variations and increase in volume fraction MS as heating was initiated (V2 and to a lesser extent V3) are an artefact of the sample structural changes at this time, they do not influence the overall analysis of the data presented elsewhere. Increased leaching caused greater bulk change in gangue distribution in the lower part of the column at this time.

At this fragment scale the initial MS volume fraction (Table 2) ranges from $0.25 \%$ to $6.23 \%$ for the typical regions and from $13.04 \%$ to $61.55 \%$ for the liberated grains. The fragment-based volume fraction reductions range from $21.99 \%$ to $98.94 \%$ (except for two of the liberated grains which showed reductions $<10 \%$ ), and the majority of the regions showed in excess of $50 \%$ reduction in MS volume fraction (Table 2). There is no relationship between the reduction and the initial MS content of the total fragment volume. The largest fragments $(3,13$, and 25; Table 2) show some of the lowest volume reductions, but the reductions in the smaller fragments show no clear volume dependence. This suggests that a simple shrinking core mechanism is a poor model of the behaviour, and that the penetration of the leachate into the fragments is spatially variable. A qualitative assessment of fragment specific features such as MS distribution, overall MS grain size, occurrence of veins or atypically high porosity also apparently have little influence on the overall reduction in MS volume (Table 2), although full quantification of the features is beyond the scope of this study. Detailed analysis of leaching efficiency with distance from the particle boundary [21], performed on a different agglomerated column from same deposit suggests preferentially leaching of MS grains within a $2 \mathrm{~mm}$ annulus of the particle boundary. Fragments with similar MS volume fractions show a greater difference in behaviour within a single column volume (e.g., 5 and 7; Table 2) than they do along the column (variability between V2 and V3 is same as within V1 for all fragments with MS $<1 \%$ ), and the range of leaching efficacies along the column is also constant (V1: $22.6 \%$ to $83.0 \%$ reduction, $\mathrm{V} 2: 22.0 \%$ to $98.9 \%$ reduction, V3: $35.2 \%$ to $96.6 \%$ reduction). 
Table 2. Volumetric changes observed.

\begin{tabular}{|c|c|c|c|c|c|c|c|c|c|c|c|c|c|c|c|c|c|c|c|c|c|c|c|}
\hline \multirow{2}{*}{ \# } & \multirow{2}{*}{ Type } & \multirow{2}{*}{$\begin{array}{l}\text { Frag } \\
\text { vol }^{*} \\
(\%)\end{array}$} & \multirow{2}{*}{$\underset{\mu \mathrm{m}}{\text { Radii }}$} & \multirow{2}{*}{$\begin{array}{c}\text { MS } \\
\text { vol } \\
\% \\
\end{array}$} & \multirow{2}{*}{$\begin{array}{c}\text { MS vol } \\
\% \\
\text { (D192) }\end{array}$} & \multirow{2}{*}{$\begin{array}{c}\text { MS } \\
\text { Reduct. } \\
(\%)\end{array}$} & \multirow{2}{*}{$\begin{array}{c}A \\
\text { (D0) } \\
\%\end{array}$} & \multirow{2}{*}{$\begin{array}{c}d A \\
\text { (D0) } \\
\%\end{array}$} & \multirow{2}{*}{$\begin{array}{l}i d A \\
\text { (D0) } \\
\%\end{array}$} & \multirow{2}{*}{$\begin{array}{c}n A \text { (D0) } \\
\%\end{array}$} & \multirow{2}{*}{$\begin{array}{c}A \\
\text { (D192) } \\
\%\end{array}$} & \multirow{2}{*}{$\begin{array}{c}d A \\
\text { (D192) } \\
\%\end{array}$} & \multirow{2}{*}{$\begin{array}{c}i d A \\
\text { (D192) } \\
\%\end{array}$} & \multirow{2}{*}{$\begin{array}{c}n A \\
\text { (D192) } \\
\%\end{array}$} & \multirow{2}{*}{$\begin{array}{c}\Delta d A \\
\text { vol } \\
\%\end{array}$} & \multirow{2}{*}{$\begin{array}{c}\% \\
\text { Reduct. }\end{array}$} & \multirow{2}{*}{$\begin{array}{c}\Delta \text { idA } \\
\text { vol } \\
\% \\
\end{array}$} & $\%$ & $\Delta n A$ & $\%$ & Total & Recove & $y(\%)$ \\
\hline & & & & & & & & & & & & & & & & & & Reduct. & $\begin{array}{c}\text { vol } \\
\%\end{array}$ & Reduct. & $d A$ & idA & $n A$ \\
\hline Fraq & nents in & 11 (top) & & (D0) & & & & & & & & & & & & & & & & & & & \\
\hline 1 & $1 b c$ & 4.42 & 1551 & 0.25 & 0.08 & 69.5 & 0.15 & 0.06 & 0.09 & 0.05 & 0.06 & 0.01 & 0.05 & 0.01 & 0.05 & 77.7 & 0.04 & 44.7 & 0.03 & 74.7 & 40.4 & 31.9 & 27.8 \\
\hline 2 & $1 e^{f, 2}$ & 1.22 & 1551 & 1.10 & 0.22 & 79.6 & 0.52 & 0.26 & 0.25 & 0.40 & 0.17 & 0.04 & 0.12 & 0.00 & 0.22 & 84.3 & 0.13 & 51.2 & 0.40 & 100 & 29.3 & 17.2 & 53.5 \\
\hline 3 & $2 c$ & 0.27 & 1056 & 6.12 & 3.95 & 35.4 & 4.88 & 0.50 & 4.38 & 1.19 & 3.14 & 0.13 & 3.01 & 0.81 & 0.37 & 74.0 & 1.37 & 31.2 & 0.38 & 31.8 & 17.6 & 64.6 & 17.9 \\
\hline 4 & $1 b c e^{c}$ & 2.54 & 2244 & 0.66 & 0.11 & 83.2 & 0.14 & 0.07 & 0.07 & 0.37 & 0.08 & 0.03 & 0.06 & 0.03 & 0.05 & 62.9 & 0.01 & 19.1 & 0.35 & 93.0 & 11.2 & 3.4 & 85.5 \\
\hline 5 & $1 b c d$ & 4.34 & 2244 & 0.59 & 0.28 & 52.2 & 0.33 & 0.16 & 0.17 & 0.17 & 0.18 & 0.05 & 0.13 & 0.10 & 0.11 & 69.4 & 0.04 & 21.4 & 0.08 & 43.5 & 50.0 & 16.2 & 33.8 \\
\hline 6 & $1 b$ & 10.79 & 3168 & 0.30 & 0.12 & 61.3 & 0.16 & 0.07 & 0.09 & 0.10 & 0.10 & 0.02 & 0.08 & 0.02 & 0.05 & 69.7 & 0.01 & 15.6 & 0.08 & 81.6 & 32.5 & 9.9 & 57.6 \\
\hline 7 & $2 b c$ & 8.95 & 2772 & 0.49 & 0.15 & 68.7 & 0.18 & 0.08 & 0.10 & 0.20 & 0.10 & 0.02 & 0.08 & 0.05 & 0.06 & 73.5 & 0.02 & 21.4 & 0.16 & 75.8 & 24.9 & 9.4 & 65.7 \\
\hline 8 & $1 a$ & 2.72 & 1419 & 2.79 & 0.67 & 75.9 & 1.73 & 0.46 & 1.27 & 0.83 & 0.56 & 0.09 & 0.47 & 0.11 & 0.37 & 80.3 & 0.80 & 63.3 & 0.72 & 86.4 & 19.6 & 42.4 & 38.0 \\
\hline 9 & $1 b d$ & 14.40 & 2574 & 1.69 & 0.78 & 53.6 & 0.61 & 0.17 & 0.45 & 0.83 & 0.36 & 0.04 & 0.31 & 0.43 & 0.12 & 74.3 & 0.13 & 29.8 & 0.40 & 48.4 & 18.9 & 20.1 & 61.0 \\
\hline 10 & $2 b c$ & 21.04 & 3498 & 3.52 & 2.72 & 22.6 & 0.70 & 0.12 & 0.58 & 2.46 & 0.42 & 0.03 & 0.39 & 2.30 & 0.09 & 75.3 & 0.19 & 33.0 & 0.16 & 6.4 & 21.0 & 43.2 & 35.8 \\
\hline Frą & nents in & 22 (midd & & & & & & & & & & & & & & & & & & & & & \\
\hline 11 & $1 b c$ & 0.98 & 1419 & 1.27 & 0.07 & 94.7 & 0.61 & 0.27 & 0.33 & 0.66 & 0.05 & 0.02 & 0.03 & 0.02 & 0.25 & 92.4 & 0.30 & 90.8 & 0.64 & 97.6 & 21.1 & 25.3 & 53.6 \\
\hline 12 & $1 b c$ & 2.95 & 1947 & 0.49 & 0.01 & 98.6 & 0.39 & 0.16 & 0.23 & 0.10 & 0.00 & 0.00 & 0.00 & 0.00 & 0.16 & 98.5 & 0.23 & 99.9 & 0.09 & 95.8 & 32.8 & 47.8 & 19.4 \\
\hline 13 & $1 b e^{f}$ & 1.60 & 1848 & 1.14 & 0.01 & 98.9 & 0.28 & 0.14 & 0.14 & 0.86 & 0.00 & 0.00 & 0.00 & 0.01 & 0.14 & 100 & 0.14 & 99.7 & 0.84 & 98.6 & 12.8 & 12.1 & 75.1 \\
\hline 14 & $1 a$ & 2.99 & 2541 & 1.19 & 0.05 & 96.1 & 0.55 & 0.23 & 0.32 & 0.65 & 0.03 & 0.01 & 0.01 & 0.02 & 0.22 & 94.5 & 0.30 & 95.4 & 0.63 & 97.1 & 19.0 & 26.2 & 54.9 \\
\hline 15 & $1 c e^{c}$ & 3.43 & 1947 & 4.43 & 1.40 & 68.3 & 2.41 & 0.23 & 2.17 & 2.02 & 0.02 & 0.00 & 0.01 & 1.39 & 0.23 & 98.3 & 2.16 & 99.4 & 0.63 & 31.3 & 7.6 & 71.5 & 21.0 \\
\hline 16 & $1 a c$ & 39.3 & 5214 & 4.19 & 3.27 & 22.0 & 1.42 & 0.14 & 1.28 & 2.77 & 0.42 & 0.01 & 0.41 & 2.85 & 0.14 & 93.6 & 0.87 & 67.8 & -0.08 & -2.9 & 14.7 & 94.0 & -8.7 \\
\hline & nents in & 3 (botto & & & & & & & & & & & & & & & & & & & & & \\
\hline 17 & $1 a$ & 2.25 & 2244 & 0.33 & 0.01 & 96.6 & 0.12 & 0.09 & 0.02 & 0.21 & 0.00 & 0.00 & 0.00 & 0.01 & 0.09 & 99.2 & 0.02 & 95.1 & 0.20 & 95.5 & 29.74 & 7.03 & 63.23 \\
\hline 18 & $1 a$ & 0.55 & 1617 & 2.83 & 1.35 & 52.4 & 1.51 & 0.52 & 0.99 & 1.32 & 0.50 & 0.09 & 0.41 & 0.84 & 0.42 & 81.8 & 0.59 & 58.9 & 0.48 & 36.1 & 28.43 & 39.44 & 32.13 \\
\hline 19 & $1 c$ & 1.61 & 1914 & 1.59 & 0.59 & 62.9 & 0.56 & 0.27 & 0.29 & 1.03 & 0.02 & 0.02 & 0.00 & 0.57 & 0.25 & 92.9 & 0.29 & 98.9 & 0.46 & 44.9 & 24.88 & 28.85 & 46.27 \\
\hline 20 & $1 b$ & 2.99 & 1782 & 1.25 & 0.17 & 86.2 & 0.50 & 0.14 & 0.36 & 0.75 & 0.03 & 0.01 & 0.02 & 0.14 & 0.14 & 93.6 & 0.34 & 94.0 & 0.60 & 81.0 & 12.57 & 31.39 & 56.04 \\
\hline 21 & $1 b, d$ & 3.98 & 1947 & 1.24 & 0.18 & 85.4 & 0.43 & 0.20 & 0.24 & 0.81 & 0.06 & 0.03 & 0.03 & 0.12 & 0.16 & 83.8 & 0.21 & 87.9 & 0.69 & 85.0 & 15.51 & 19.61 & 64.88 \\
\hline 22 & $1 a$ & 4.60 & 2079 & 1.38 & 0.89 & 35.2 & 0.45 & 0.21 & 0.25 & 0.93 & 0.18 & 0.07 & 0.12 & 0.71 & 0.14 & 67.6 & 0.13 & 52.7 & 0.22 & 23.3 & 28.96 & 26.57 & 44.47 \\
\hline 23 & $1 b$ & 5.01 & 2607 & 1.41 & 0.17 & 88.2 & 0.63 & 0.13 & 0.50 & 0.78 & 0.03 & 0.01 & 0.02 & 0.14 & 0.12 & 93.5 & 0.48 & 96.1 & 0.64 & 82.3 & 9.75 & 38.89 & 51.35 \\
\hline 24 & $1 a$ & 2.61 & 1650 & 3.75 & 0.35 & 90.8 & 1.77 & 0.37 & 1.40 & 1.98 & 0.1 & 0.04 & 0.08 & 0.23 & 0.33 & 90.4 & 1.32 & 94.0 & 1.75 & 88.5 & 9.75 & 38.68 & 51.57 \\
\hline 25 & $1 c, 2 c$ & 4.60 & 2046 & 6.23 & 1.16 & 81.4 & 2.47 & 0.51 & 1.96 & 3.75 & 0.07 & 0.04 & 0.04 & 1.09 & 0.48 & 92.8 & 1.92 & 98.2 & 2.67 & 71.0 & 9.40 & 37.98 & 52.62 \\
\hline 26 & $1 a$ & 43.28 & 5907 & 5.32 & 3.31 & 37.8 & 0.84 & 0.14 & 0.70 & 4.47 & 0.08 & 0.02 & 0.07 & 3.22 & 0.12 & 86.6 & 0.64 & 90.7 & 1.25 & 28.0 & 6.02 & 31.73 & 62.25 \\
\hline Lib & ated Ms & rrains (a & ross all th & ree volu & & & & & & & & & & & & & & & & & & & \\
\hline 1 & 3 & 0.06 & 693 & 13.04 & 9.41 & 27.9 & 12.98 & 2.71 & 10.27 & 0.13 & 9.40 & 0.67 & 8.74 & 0.00 & 2.04 & 75.4 & 1.54 & 15.0 & 0.13 & 100 & 55.05 & 41.45 & 3.50 \\
\hline 2 & 3 & 0.08 & 792 & 42.21 & 38.01 & 9.9 & 42.67 & 8.87 & 33.80 & 0.00 & 38.01 & 3.10 & 34.90 & 0.00 & 5.77 & 65.0 & -1.11 & -3.3 & 0.00 & $n / a$ & 123.73 & 23.73 & $n / a$ \\
\hline 3 & 3 & 0.11 & 726 & 61.55 & 11.28 & 81.7 & 61.55 & 12.08 & 49.47 & 0.00 & 11.28 & 1.39 & 9.88 & 0.00 & 10.69 & 88.5 & 39.58 & 80.0 & 0.00 & $n / a$ & 21.26 & 78.74 & $n / a$ \\
\hline 4 & 3 & 0.06 & 396 & 38.58 & 1.97 & 94.9 & 38.33 & 11.50 & 26.83 & 0.25 & 1.73 & 0.52 & 1.21 & 0.24 & 10.98 & 95.5 & 25.62 & 95.5 & 0.01 & 2.84 & 29.99 & 69.99 & 0.02 \\
\hline 5 & 3 & 0.07 & 330 & 57.40 & 4.33 & 92.5 & 57.39 & 17.79 & 39.60 & 0.00 & 3.99 & 2.27 & 1.72 & 0.00 & 15.52 & 87.2 & 37.88 & 95.7 & 0.00 & $n / a$ & 29.07 & 70.93 & $n / a$ \\
\hline 6 & 3 & 0.07 & 528 & 45.03 & 44.33 & 1.6 & 45.02 & 9.87 & 35.15 & 0.00 & 44.33 & 9.34 & 34.99 & 0.00 & 0.54 & 5.4 & 0.16 & 0.6 & 0.00 & $n / a$ & 77.05 & 22.95 & $n / a$ \\
\hline
\end{tabular}




\section{Quantification of Ore Exposure}

The 3D images reveal the complexity in the spatial distribution of volume reduction. The volume of accessible MS grains (grains exposed on the surface) is widely used to assess the potential recovery from an ore. For V1-V3, a binary map of the external connected "leachate accessible" porosity was used to calculate the Euclidian distance between every ore voxel and the nearest ore-pore interface. A filter was applied to the resultant distance maps to generate a mask containing all the voxels less than 3 voxels from the pore-ore interface, and that therefore have face, edge or corner contact with the pore volume. These voxels are defined as directly accessible to the external pore volume (gold, Figure 6). This definition allows for roughness in the ore surface which is beyond the spatial resolution of the images. Each MS voxel was defined as either being directly, indirectly or not accessible (Table 1). Directly accessible MS voxels are within the surface region defined above; indirectly accessible voxels are part of MS grains that have contact with an accessible MS voxel (red, Figure 6). We can then investigate the relationship between the changing MS grain volume and sulfide accessibility; testing the assumptions regarding accessibility and leaching.

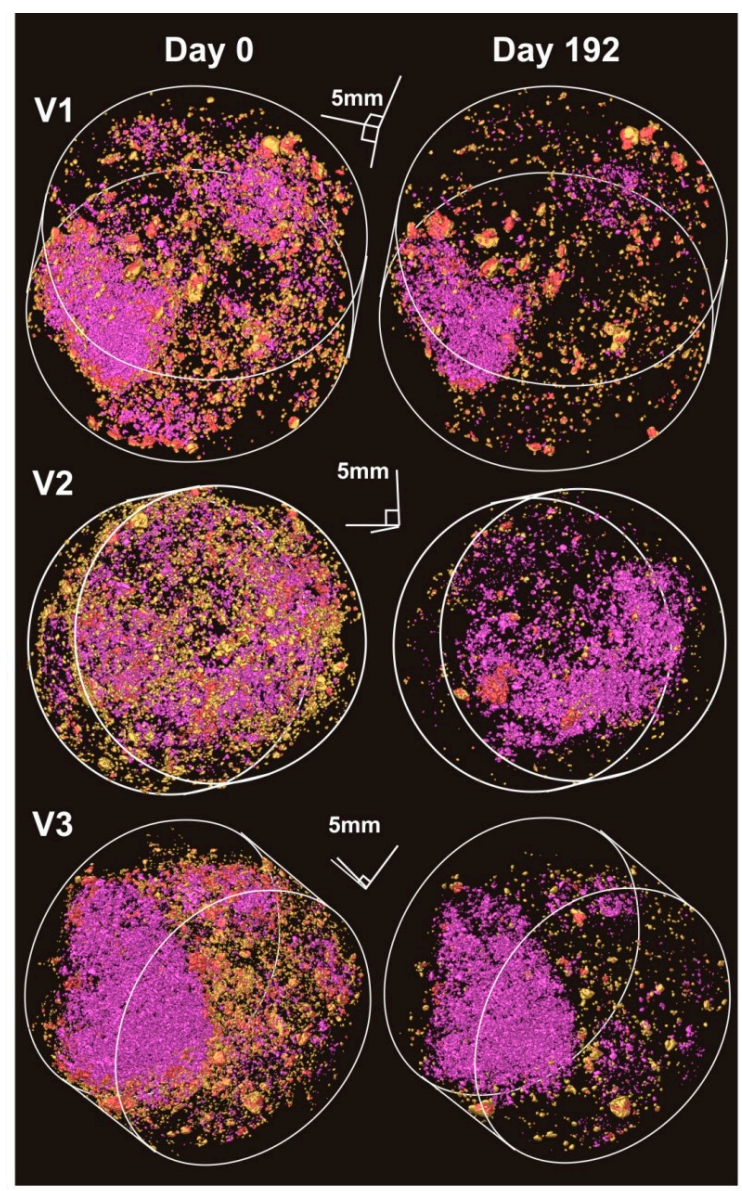

Figure 6. 3D render showing the accessibility of the leached grains at Day 0 and Day 192. Gold—directly accessible; Red—indirectly accessible; Purple—not accessible. The gangue has been rendered invisible. Only MS grains with volume $>5$ voxels are shown. The surfaces have been down sampled by a factor of 2 in all dimensions to improve clarity of the figure. The outline of the column volume is shown in white. All scale bars are $5 \mathrm{~mm}$.

\subsection{Volume Scale Behaviours}

Prior to leaching $~ 53 \%$ of the MS grains ( $0.87 \mathrm{vol} \%$ ) in V1, $45 \%$ of the MS grains in V2 (1.38 vol \%), and $35 \%$ of the MS grains in V3 (1.29 vol \%) are accessible (Table 1). After leaching the volume of 
accessible MS has reduced significantly, but this reduction in V1 is substantially lower ( $43 \%$ reduction) than in V2 and V3 ( $84 \%$ reduction). The volume of non-accessible MS has also reduced by between $23 \%$ (V1 and V2) and 35\% (V3). V2 and V3 show very similar leaching behaviour with very efficient leaching in both the directly and indirectly accessible regions and less efficient leaching of non-accessible MS. When considering the location on the leaching, the greatest contribution to the overall reduction comes from the indirectly accessible MS in V1 and V2 and from the directly accessible MS in V3, correlating to the regions of the ore with the highest MS content.

Large non-leached liberated MS grains will strongly bias the directly and indirectly accessible measurements; however, these are relatively evenly distributed through the column and so are unlikely to account for the observed differences between the volume behaviours. Without a better constraint on the spatial distribution of the different MS phases before and after leaching, further interpretation of this data is difficult; however, our numerical approach suggests that simple accessibility in terms of access through porosity at the scale used in this analysis is not the only defining parameter for the prediction of recovery of the leachable fraction from real ores. This may partly be complicated by the differing leachability of the different mineral sulphides present as well as by characteristics of the individual mineral grains.

\subsection{Fragment Scale Behaviours}

Applying the region masks to the directly, indirectly and non-accessible MS voxel maps shows that volume scale quantification obscures the detail of fragment scale behaviours. Minimum reductions range from $63 \%$ (directly accessible) to $0 \%$ (non-accessible), and complete removal of all MS is observed from all regions at least one fragment. For fragments with similar initial MS content, volume reductions can range from $35 \%$ to $88 \%$. For two particular fragments (22 and 23; Table 2) with mid-range initial MS ( 1.4 vol \%), the larger fragment has the higher leaching efficacy. On first inspection, there is no reduction in leaching efficacy with increasing fragment volume (Figure 7); however, the calculated volume of each of the fragments does not assess fragment geometry, and for the highly irregular fragments in this agglomerated ore, the fragment volume does not assess total leachate penetration distance. Further, variation in the leaching kinetics of the metal sulfides present is well accepted, implying that a differential analysis of these sulfides would be necessary for rigorous assessment of the leaching mechanism.

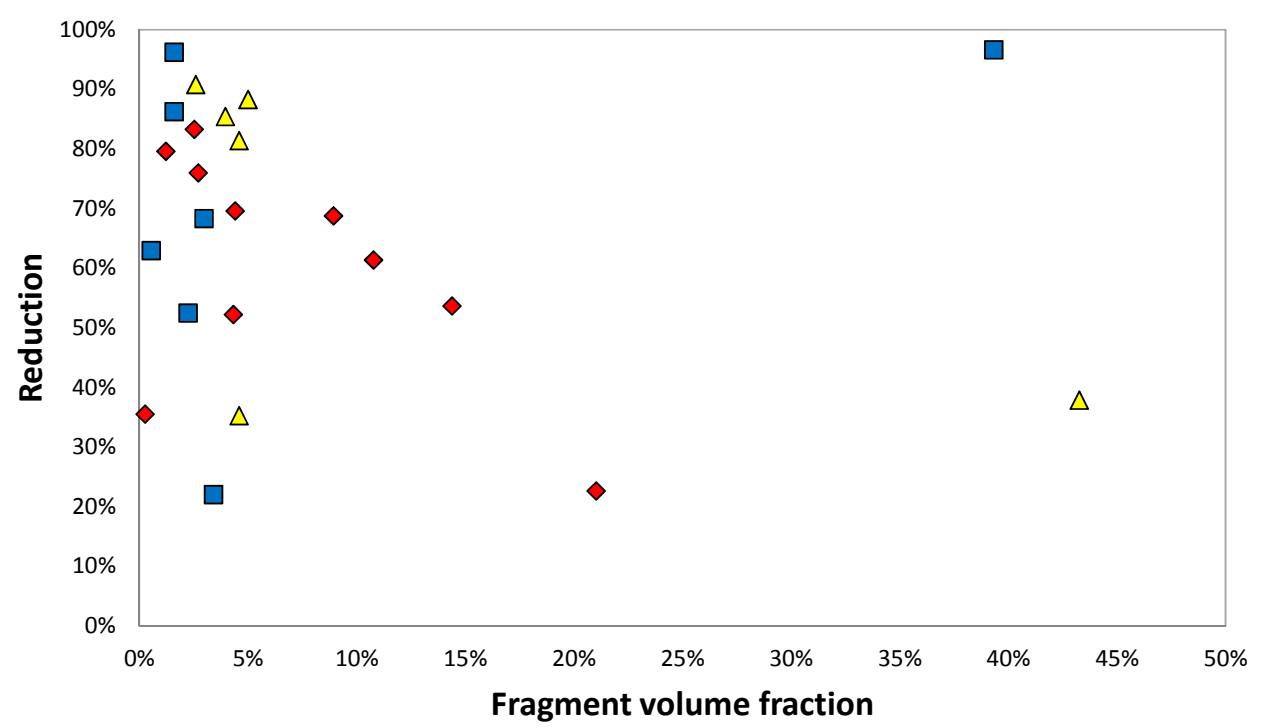

Figure 7. Fragment scale leaching behaviour showing the lack of correlation between fragment volume and fragment scale volume fraction MS reduction for V1 (red diamonds), V2 (blue squares) and V3 (yellow triangles). Only MS grains with volume $>5$ voxels are included in the calculations. 


\section{Spatial Heterogeneity in MS Leaching Behaviour}

Our analysis shows that the large-scale volume averaging used in quantification on the column and volume scale obscures significant spatial heterogeneity in MS volume reduction. The heterogeneity implies that better understanding of the mechanisms controlling leaching efficacy and quantification of the behaviours on the $\mathrm{mm}^{3}$ to $\mathrm{cm}^{3}$ scale is required. The increased efficiency of leaching at lower positions in the column (V3) suggests that consumption of the reagents as the solution percolates through the column was sufficiently small not to affect the leaching according to the desired experimental design of this small-scale system; hence consistent leaching could be expected in the axial direction down the column, and is confirmed by the very small change in $\mathrm{pH}$ and redox potential of the feed irrigant and effluent confirming abundance of protons and ferric iron as leach agents throughout the column. Excessive reagent consumption (not seen) would decrease the total volume reduction down-column, and/or cause a temporal offset; with leaching initially occurring at the top of the column and a reaction front progressing downward as $\mathrm{Cu}$ extraction is completed. The data from this column does not show a clear offset in the progression of leaching (Figure 5), supporting the provision of excess leach agent throughout the column. The distribution of the leaching probably reflects the widening lateral dispersion of the reagent as it percolates downward [30], potential wall effects and the presence of rivulets of irrigant $[29,31]$ Between V1 and V2 one very oblate fragment extends across most of the column cross sectional area (see Figure 2), and could generate significant flow disruption. The geometry of the fragment may generate multiple drip points and a higher density of flow rivulets through the lower section of the column.

\section{Conclusions}

A lack of understanding of the $\mathrm{mm}^{3}$ to $\mathrm{cm}^{3}$ controls on leaching from ore fragments under heap conditions limits the development of predictive models that can be used to improve total $\mathrm{Cu}$ recovery and heap efficiency. Existing models generally fail to incorporate leaching behaviour variability across different length scales because they have insufficient experimentally-derived information to link macro and micro scale understanding. Using a $\mathrm{Cu}$-sulfide bearing low-grade agglomerated ore we have used X-ray computed tomography to provide a detailed analysis of the magnitude of the spatial heterogeneity in the sulfide distribution in the agglomerated ore, the magnitude of the volume reduction, and the spatial distribution of that leaching in relation to the accessibility of the sulfide grains. This suggests that at the $\mathrm{mm}^{3}$ to $\mathrm{cm}^{3}$ scale, leaching does not necessarily follow a simple shrinking core model. We applied a novel in situ, non-destructive form of the X-ray-computed tomography analytical method to perform high-resolution time series analysis of small-scale experimental leaching columns. This has enabled interrogation of the leaching controls that operate at the crucial intermediate length scale. Information provided by further investigation will deliver the necessary experimental parameters for better heap leaching models, and ultimately feed into developing better ore and heap specific leaching protocols.

Acknowledgments: We would like to acknowledge the assistance provided by the Manchester X-ray Imaging Facility in the Research Complex at Harwell, funded in part by the EPSRC (EP/I02249X/1). STLH acknowledges the South African Research Chairs Initiative of the Department of Science and Technology (NRF UID 64778) for their sponsorship and support of this project.

Author Contributions: P.D.L., J.J.C., and S.T.L.H. conceived the study and designed the experiments; A.N.B., S.T.L.H. and M.A.F.E. performed the experiments; K.J.D. performed the analysis and led the writing of the paper with input from P.D.L., J.J.C., S.T.L.H., Q.L., M.A.F.E. and S.J.N.

Conflicts of Interest: The authors declare no conflict of interest. The founding sponsors had no role in the design of the study; in the collection, analyses, or interpretation of data; in the writing of the manuscript, and in the decision to publish the results. 


\section{References}

1. Kotsiopouios, A.; Harrrison, S.T.L. Application of fine desulfurised coal tailings as a neutralising barrier in the prevention of acid rock drainage. Hydrometallurgy 2017, 168, 159-166. [CrossRef]

2. Bartlett, R.W. Metal extraction from ores by heap leaching. Metall. Mater. Trans. B Process Metall. Mater. Process. Sci. 1997, 28, 529-545. [CrossRef]

3. Brierley, J.A. A perspective on developments in biohydrometallurgy. Hydrometallurgy 2008, 94, 2-7. [CrossRef]

4. Petersen, J.; Dixon, D.G. Modelling zinc heap bioleaching. Hydrometallurgy 2007, 85, 127143. [CrossRef]

5. Watling, H.R. The bioleaching of nickel-copper sulfides. Hydrometallurgy 2008, 91, 70-88. [CrossRef]

6. Watling, H.R. The bioleaching of sulphide minerals with emphasis on copper sulphides-A review. Hydrometallurgy 2006, 84, 81-108. [CrossRef]

7. Leahy, M.J.; Schwarz, M.P. Modelling jarosite precipitation in isothermal chalcopyrite bioleaching columns. Hydrometallurgy 2009, 98, 181-191. [CrossRef]

8. Breed, A.W.; Hansford, G.S. Modelling continuous bioleach reactors. Biotechnol. Bioeng. 1999, 64, 671-677. [CrossRef]

9. Breed, A.W.; Hansford, G.S. Studies on the mechanism and kinetics of bioleaching. Miner. Eng. 1999, 12, 383-392. [CrossRef]

10. Cordoba, E.M.; Muñozb, J.A.; Blázquezb, M.L.; Gonzálezb, F.; Ballesterb, A. Leaching of chalcopyrite with ferric ion. Part I: General aspects. Hydrometallurgy 2008, 93, 81-87. [CrossRef]

11. Ketcham, R.A.; Carlson, W.D. Acquisition, optimization and interpretation of X-ray computed tomographic imagery: applications to the geosciences. Comput. Geosci. 2001, 27, 381-400. [CrossRef]

12. Stock, S.R. Recent advances in X-ray microtomography applied to materials. Int. Mater. Rev. 2008, 53, 129-181. [CrossRef]

13. Lin, C.L.; Miller, J.D. 3D characterization and analysis of particle shape using X-ray microtomography (XMT). Powder Technol. 2005, 154, 61-69. [CrossRef]

14. Ghorbani, Y.; Becker, M.; Mainza, A.; Franzidis, J.P.; Petersen, J. Large particle effects in chemical/biochemical heap leach processes-A review. Miner. Eng. 2011, 24, 1172-1184. [CrossRef]

15. Ghorbani, Y.; Becker, M.; Petersen, J.; Morar, S.H.; Mainza, A.; Franzidis, J.-P. Use of X-ray computed tomography to investigate crack distribution and mineral dissemination in sphalerite ore particles. Miner. Eng. 2011, 24, 1249-1257. [CrossRef]

16. Kodali, P.; Dhawan, N.; Depci, T.; Lin, C.L.; Miller, J.D. Particle damage and exposure analysis in HPGR crushing of selected copper ores for column leaching. Miner. Eng. 2011, 24, 1478-1487. [CrossRef]

17. Miller, J.D.; Lin, C.L.; Garcia, C.; Arias, H. Ultimate recovery in heap leaching operations as established from mineral exposure analysis by X-ray microtomography. Int. J. Miner. Process. 2003, 72, 331-340. [CrossRef]

18. Ghorbani, Y.; Mainza, A.N.; Petersen, J.; Becker, M.; Franzidis, J.P.; Kalala, J.T. Investigation of particles with high crack density produced by HPGR and its effect on the redistribution of the particle size fraction in heaps. Miner. Eng. 2013, 43-44, 44-51. [CrossRef]

19. Lin, Q.; Barker, D.J.; Dobson, K.J.; Lee, P.D.; Neethling, S.J. Modelling particle scale leach kinetics based on X-ray computed micro-tomography images. Hydrometallurgy 2016, 162, 25-36. [CrossRef]

20. Lin, Q.; Neethling, S.; Courtois, L.; Dobson, K.; Lee, P. Multi-scale quantification of leaching performance using X-ray tomography. Hydrometallurgy 2016, 164, 265-277. [CrossRef]

21. Fagan-Endres, M.A.; Cilliers, J.J.; Sederman, A.J.; Harrison, S.T.L. Spatial variations in leaching of a low-grade, low-porosity chalcopyrite ore identified using X-ray $\mu \mathrm{CT}$. Miner. Eng. 2017, 105, 63-68. [CrossRef]

22. Schindelin, J.; Arganda-Carreras, I.; Frise, E.; Kaynig, V.; Longair, M.; Pietzsch, T.; Preibisch, S.; Rueden, C.; Saalfeld, S.; Schmid, B.; et al. Fiji: An open-source platform for biological-image analysis. Nat. Methods 2012, 9, 676-682. [CrossRef] [PubMed]

23. Africa, C.-J.; van Hille, R.P.; Sand, W.; Harrison, S.T.L. Investigation and in situ visualisation of interfacial interactions of thermophilic microorganisms with metal-sulphides in a simulated heap environment. Miner. Eng. 2013, 48, 100-107. [CrossRef]

24. Van Hille, R.P.; van Zyl, A.W.; Spurr, N.R.L.; Harrison, S.T.L. Investigating heap bioleaching: Effect of feed iron concentration on bioleaching performance. Miner. Eng. 2010, 23, 518-525. [CrossRef] 
25. Fagan, M.A.; Sederman, A.J.; Johns, M.L. MR imaging of ore for heap bioleaching studies using pure phase encode acquisition methods. J. Magn. Reson. 2012, 216, 121-127. [CrossRef] [PubMed]

26. Petersen, J. Heap leaching as a key technology for recovery of values from low-grade ores-A brief overview. Hydrometallurgy 2016, 165, 206-212. [CrossRef]

27. Harrison, S.T.L.; Stevenson, R.; Cilliers, J.J. Assessing solids concentration homogeneity in Rushton-agitated slurry reactors using electrical resistance tomography (ERT). Chem. Eng. Sci. 2012, 71, 392-399. [CrossRef]

28. Lin, Q.; Neethling, S.J.; Dobson, K.J.; Courtois, L.; Lee, P.D. Quantifying and minimising systematic and random errors in X-ray micro tomography based volume measurements. Comput. Geosci. 2015, 77, 1-7. [CrossRef]

29. Fagan, M.A.; Sederman, A.J.; Harrison, S.T.L.; Johns, M.L. Phase distribution identification in the column leaching of low grade ores using MRI. Miner. Eng. 2013, 48, 94-99. [CrossRef]

30. Fagan, M.A.; Ngoma, I.E.; Chiume, R.A.; Minnaar, S.; Sederman, A.J.; Johns, M.L.; Harrison, S.T.L. MRI and gravimetric studies of hydrology in drip irrigated heaps and its effect on the propoagation of bioleaching micro-organisms. Hydrometallurgy 2014, 150, 210-221. [CrossRef]

31. Ilankoon, I.M.S.K.; Neethling, S.J. Transient liquid holdup and drainage variations in gravity dominated non-porous and porous packed beds. Chem. Eng. Sci. 2014, 116, 398-405. [CrossRef]

(C) 2017 by the authors. Licensee MDPI, Basel, Switzerland. This article is an open access article distributed under the terms and conditions of the Creative Commons Attribution (CC BY) license (http:/ / creativecommons.org/licenses/by/4.0/). 